\title{
Editorial
}

\section{Percutaneous Endoscopic Gastrostomy: An Effective yet Underutilized Procedure in India}

\author{
Suhang Verma ${ }^{1}$ Usha Dutta ${ }^{1}$ \\ ${ }^{1}$ Department of Gastroenterology, Postgraduate Institute of \\ Medical Education and Research (PGIMER), Chandigarh, India
}

J Digest Endosc 2019;10:155-157

Maintenance of long-term nutrition poses a challenge in patients with medical disorders with inadequate oral intake. Enteral nutrition is the preferred route in patients with intact gastrointestinal tract and has multiple proven benefits over parenteral nutrition including lower risk of sepsis, maintenance of gut barrier, and microbiota. ${ }^{1}$ Stomach is the most commonly used site for long-term tube feeding when feasible. Access can be maintained by using a nasogastric tube or a percutaneous gastrostomy that may be placed surgically, radiologically, or endoscopically. ${ }^{2}$ While nasoenteric tube may be used for short-term enteral feed ( $<4$ weeks), they are associated with higher complications such as nasal/ throat irritation, reflux esophagitis, and aspiration pneumonia particularly in patients with impaired laryngeal reflexes and thus not the preferred mode for long-term nutritional needs. ${ }^{3}$ Percutaneous endoscopic gastrostomy (PEG) is the preferred mode for establishing long-term tube feeding worldwide. A single-center experience from India is published in this edition of the journal.

PEG was first described in 1980. The techniques and devices available have since further evolved to become the most patient- and gastroenterologist-friendly method., ${ }^{4,5}$ Compared with surgical feeding, gastrostomy/jejunostomy is of lower cost, requires shorter hospital stays, induces lesser pain, and does not require general anesthesia. Many of these debilitated patients are often poor candidates for general anesthesia. ${ }^{6}$ Most common indications of PEG tube placement include neurological diseases (cerebrovascular disease, motor neuron disease, dementia, prolonged coma) and malignancy (head and neck cancer, esophageal cancer). ${ }^{2}$ Expected survival of the patient should be preferably be over a month to justify the procedure. Commonly encountered contraindications to PEG tube placement include uncontrolled bleeding diathesis, hemodynamic instability, gross ascites, sepsis, peritonitis, abdominal wall infections, gastroparesis, and gastric outlet obstruction and should be excluded prior to the procedure. ${ }^{3}$
Among the three commonly available techniques: pull method, push method, and introducer method; the "pull method" is the most preferred technique. ${ }^{2}$ The procedure is usually safe. However, serious complications may occur rarely, which include-bleeding, perforation peritonitis, local site infections and necrotizing fasciitis, aspiration pneumonia, and buried bumper syndrome., ${ }^{2,7} \mathrm{~A}$ single dose of preprocedure antibiotic (usually $1 \mathrm{~g}$ cefazolin) is recommended as the standard practice to reduce infective complications. ${ }^{8}$ Postprocedure stoma and tube care is equally important to prevent complications. Stoma should be cleaned and examined for signs of infection and excoriation on a daily basis. Thick feeds should be avoided and tube should be flushed with warm water before and after each feed to prevent clogging. ${ }^{3}$ Daily examination of the wound site is essential to detect complications promptly. The caregivers need to be specifically educated about this.

While the procedure is being widely practiced globally, there is dearth of published literature of its use from India. The paper published in current issue describes a single-center experience with PEG placement in the form of a retrospective study of data collected over a 3-year period. Standard "pull method" was utilized for placement of a 24-Fr PEG tube. Overall, 76 patients underwent the procedure with a mean age of 67 years. Most common indications were neurological disorders followed by malignancies. High technical success (97.3\%) was achieved; however, all the procedures were performed by a single experienced gastroenterologist. A high disease-related mortality (56.16\%) was seen during follow-up with a short median time to death (65 days) postprocedure. This probably reflects late referral of patients in India for PEG. Patients undergoing PEG are thus having advanced disease, nutritionally depleted, and debilitated at the time of procedure. This results in overall shorter survival as well as poses challenges in conduct of the procedure. Sensitization of our neurology, oncology, and surgical colleagues can improve the referral patterns and thereby improve the outcomes.

License terms
Copyright (C2019 Society of Gastrointestinal Endoscopy of India

10.1055/s-0039-3401894

ISSN 0976-5042.
Usha Dutta, MD, DM, FACG, Masters in HRM, Department of Gastroenterology, PGIMER, Chandigarh,India 160012, (e-mail: ushadutta@gmail.com). 
Local site infection remains one of the most common major postprocedure complications (5-25\%) despite the routine use of prophylactic antibiotics., ${ }^{2,9}$ The reported rate was rather low (6.8\%) in the current study. Possibly, some minor infections and inflammation due to infection without culture positivity weren't specifically tracked/reported. Pus culture was positive in all cases and all were monomicrobial (Gramnegative organisms). All but one patient (who required early removal of PEG) could be managed conservatively. Caretaker and physicians should be sensitized for optimal daily stoma care and early detection of infective complications facilitating prompt management. Antibiotic protocols should be guided by local microbiological profiles. ${ }^{9}$ In our institution, we use $1 \mathrm{~g}$ cefazolin injection. Parenteral Augmentin, levofloxacin, may also be used if patient is sensitive to cefazolin. Though the authors found Gram-negative bugs on culture, infection by Gram-positive bugs is primarily prevented by the prophylactic preprocedural antibiotic.

Inadvertent removal of PEG tube (three patients in the current study) remains a dreaded complication in neurological patients who are often agitated, resulting in medical/ surgical emergency. While the early dislodgement rates have been reported to be low (0.6-4\%), the lifetime risk of inadvertent removal has been reported to be as high as $12 \%{ }^{10}$ The risk can be mitigated with simple measures such as use of mittens. The tube can be replaced through the same tract if the tract is mature (usually $>4$ weeks post-PEG) and patient presents within $<24$ hours. ${ }^{2}$ Otherwise, placement of a new tube requires a repeat endoscopic procedure by puncturing the same site or a nearby site.

One patient developed pneumoperitoneum while replacing an inadvertently removed PEG tube and required surgery for a feeding jejunostomy. Development of pneumoperitoneum is a common, self-limiting postprocedural finding on abdominal X-ray and does not necessitate surgical intervention unless there are features of peritonitis. It is generally not considered a complication and is managed conservatively. ${ }^{11}$ If features of peritonitis are present, cross-sectional imaging followed by radiological/surgical intervention may be required apart from institution of parenteral antibiotics, parenteral fluid infusion, keeping patient nil by mouth, and placing a nasogastric tube.

The authors have not highlighted some of the other commonly encountered complications such as tube dysfunction,

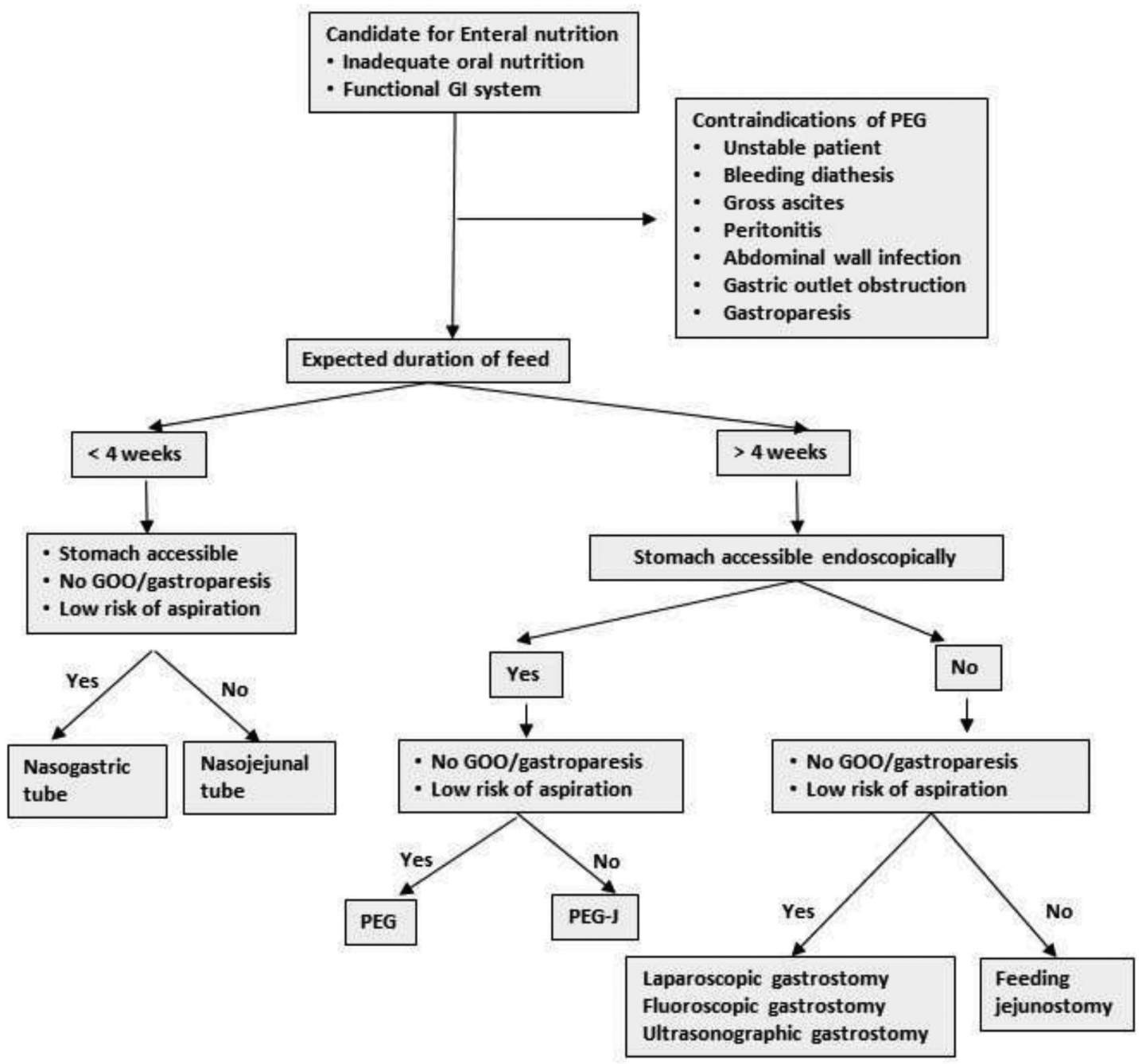

Fig. 1 Approach to patients requiring enteral nutrition therapy. 
peristomal leak, bleeding, and aspiration, among others. They have also not provided the number of patients who required tube replacements during follow-up as a result of dysfunction. Moreover, it is a single-center experience of a single trained gastroenterologist.

Despite its many advantages and safety, PEG remains an underutilized procedure in India, primarily because of the lack of awareness and willingness on the part of primary care physicians, neurologist, oncologists, and surgical colleagues. Moreover, due financial constraints, fear of a major procedure, and lack of family support, some patients' caregivers may opt for nasogastric feeding alone. Thus, many of the patients (primarily neurological and malignancies) who are candidates for long-term enteral nutrition are maintained on nasogastric tubes or undergo feeding jejunostomy rather than PEG placement. It is need of the hour to encourage timely screening and referral of suitable candidates so that an optimal route of enteral nutrition can be established. In the patients who undergo PEG placement, it is equally important to educate the caretakers regarding the optimal feeding methods and tube care so that complications such as infections, tube dysfunction, and inadvertent removal may be avoided and managed promptly.

The procedure and techniques are still evolving, and recent developments include utilizing ultrasonography, endosonography, laparoscopy, and fluoroscopy for gastrostomy tube placement in patients where stomach is not accessible endoscopically due to pharyngeal or esophageal malignancies. PEG-jejunostomy can be used to deliver postpyloric feed in patients who are at high risk for aspiration (impaired laryngeal reflexes, vocal cord palsy). A simple practical algorithm is shown in - Fig. 1.

In India, despite the procedure being shown to be effective and relatively safe, it is underutilized. It is necessary that the procedure is taught actively in a structured way to all gastroenterology trainees so that the procedure can be performed safely. They could also undergo a certification course on the side of our major conferences, to ensure competence in all aspects. Also, our neurology, oncology, and surgical colleagues need to be sensitized regarding the safety and effectiveness of the procedure so that more patients may benefit. To keep a check on cost, the accessories required could be indigenously manufactured. However, quality control is essential to prevent procedural failures. Patients and their relatives may be informed by educational videos about care of the PEG tube to prevent complications. A multipronged intervention in these lines is likely to benefit the ailing patients of the country who are often either too ill to speak for themselves or cannot speak due to their neurological status.

\section{Conflict of Interest}

None declared.

\section{References}

1 Seres DS, Valcarcel M, Guillaume A. Advantages of enteral nutrition over parenteral nutrition. Therap Adv Gastroenterol 2013;6(2):157-167

2 Rahnemai-Azar AA, Rahnemaiazar AA, Naghshizadian R, Kurtz A, Farkas DT. Percutaneous endoscopic gastrostomy: indications, technique, complications and management. World J Gastroenterol 2014;20(24):7739-7751

3 Löser C, Aschl G, Hébuterne X, et al. ESPEN guidelines on artificial enteral nutrition-percutaneous endoscopic gastrostomy (PEG) Clin Nutr 2005;24(5):848-861

4 Gauderer MW, Ponsky JL, Izant RJ Jr. Gastrostomy without laparotomy: a percutaneous endoscopic technique. J Pediatr Surg 1980;15(6):872-875

5 Blumenstein I, Shastri YM, Stein J. Gastroenteric tube feeding: techniques, problems and solutions. World J Gastroenterol 2014;20(26):8505-8524

6 Pearce CB, Duncan HD. Enteral feeding. Nasogastric, nasojejunal, percutaneous endoscopic gastrostomy, or jejunostomy: its indications and limitations. Postgrad Med J 2002;78(918):198-204

7 Naik RP, Joshipura VP, Patel NR, Haribhakti SP. Complications of PEG-prevention and management. Trop Gastroenterol 2009;30(4):186-194

8 Sharma VK, Howden CW. Meta-analysis of randomized, controlled trials of antibiotic prophylaxis before percutaneous endoscopic gastrostomy. Am J Gastroenterol 2000;95(11):3133-3136

9 Vizhi K, Rao HB, Venu RP. Percutaneous endoscopic gastrostomy site infections-Incidence and risk factors. Indian J Gastroenterol 2018;37(2):103-107

10 Rosenberger LH, Newhook T, Schirmer B, Sawyer RG. Late accidental dislodgement of a percutaneous endoscopic gastrostomy tube: an underestimated burden on patients and the health care system. Surg Endosc 2011;25(10):3307-3311

11 Schrag SP, Sharma R, Jaik NP, et al. Complications related to percutaneous endoscopic gastrostomy (PEG) tubes. A comprehensive clinical review. J Gastrointestin Liver Dis 2007;16(4):407-418 